\title{
Congenital rubella still a public health problem in Italy: analysis of national surveillance data from 2005 to 2013
}

C Giambi (cristina.giambi@iss.it) ${ }^{1}$, A Filia ${ }^{1}$, M C Rota $^{1}$, M Del Manso ${ }^{1}$, S Declich ${ }^{1}$, G Nacca ${ }^{1}$, E Rizzuto ${ }^{2}$, A Bella ${ }^{1}$, regional contact points for rubella ${ }^{3}$

1. Communicable Disease Epidemiology Unit, National Centre for Epidemiology, Surveillance and Health Promotion, Istituto Superiore di Sanità, Rome, Italy

2. Communicable Diseases Unit, Directorate General of Health Prevention, Ministry of Health, Rome, Italy

3. The contact points are listed at the end of the article.

Giambi C, Filia A, Rota MC, Del Manso M, Declich S, Nacca G, Rizzuto E, Bella A, regional contact points for rubella. Congenital rubella still a public health problem in Italy: analysis of national surveillance data from 2005 to 2013. Euro Surveill. 2015;20(16):pii=21103. Available online: http://www.eurosurveillance.org/

ViewArticle.aspx?Articleld $=21103$

In accordance with the goal of the World Health Organization Regional Office for Europe, the Italian national measles and rubella elimination plan aims to reduce the incidence of congenital rubella cases toless than one case per 100,000 live births by the end of 2015. We report national surveillance data for congenital rubella and rubella in pregnancy from 2005 to 2013. A total of 75 congenital rubella infections were reported; the national annual mean incidence was 1.5/100,000 live births, including probable and confirmed cases according to European Union case definition. Two peaks occurred in 2008 and 2012 (5.0 and 3.6/100,000 respectively). Overall, 160 rubella infections in pregnancy were reported; 69/148 women were multiparous and $38 / 126$ had had a rubella antibody test before pregnancy. Among reported cases, there were 62 infected newborns, 31 voluntary abortions, one stillbirth and one spontaneous abortion. A total of 24 newborns were unclassified and 14 women were lost to follow-up, so underestimation is likely. To improve follow-up of cases, systematic procedures for monitoring infected mothers and children were introduced in 2013. To prevent congenital rubella, antibody screening before pregnancy and vaccination of susceptible women, including post-partum and post-abortum vaccination, should be promoted. Population coverage of two doses of measles-mumps-rubella vaccination of $\geq 95 \%$ should be maintained and knowledge of health professionals improved.

\section{Introduction}

Rubella is an acute contagious viral illness; if contracted early in pregnancy, it can spread from the mother to her developing baby and result in miscarriage, stillbirth or severe birth defects including deafness, blindness, cataracts, heart defects and mental retardation (congenital rubella). The risk of fetal malformation varies according to the time of onset of maternal infection and is estimated to be $90 \%$ for infants born to women infected within the first 10 weeks of pregnancy [1].
Rubella infection can be prevented by a safe and effective vaccine and the main aim of rubella control programmes is to prevent infection in pregnant women. In accordance with the objectives of the World Health Organization (WHO) Regional Office for Europe [2], the Italian national measles and rubella elimination plan aims to eliminate rubella (incidence to less than one case per 1,000,000 live births) and reduce the incidence of congenital rubella cases to less than one case per 100,000 live births by the end of 2015 [3].

Congenital rubella prevention relies on maintaining high levels of immunity ( $\geq 95 \%$ ) in the general population and on identifying and immunising susceptible women of childbearing age. This strategy allowed the elimination of rubella in the WHO Region of the Americas, where the last confirmed cases of endemic rubella and congenital rubella syndrome (CRS) were reported in 2009 [4].

In Italy, a monovalent rubella vaccine was first available in 1972; vaccination was initially recommended only for adolescent females. The monovalent vaccine was replaced in the early 1990 s by the combined measles-mumps-rubella (MMR) vaccine and in 1999 universal vaccination with one dose of MMR vaccine was included in the national immunisation programme. In 2003, when Italy approved the first national measles and congenital rubella elimination plan, a two-dose schedule was adopted in all regions. Currently, a first dose of MMR vaccine is recommended at the age of 12 to 15 months and a second dose at 5 to 6 years of age. MMR vaccination is also offered free of charge to all susceptible adolescents and adults [5].

Uptake of one dose of MMR vaccine remained below 80\% until 2002; uptake increased after implementation of the first national elimination plan (2003-07) and national vaccination coverage assessed in children at 24 months of age was $88 \%$ in 2013 [6]. Immunisation coverage of adolescents and adults is not routinely 


\section{FIGURE 1}

Notification flow for congenital rubella and rubella infections in pregnancy in Italy

Who?

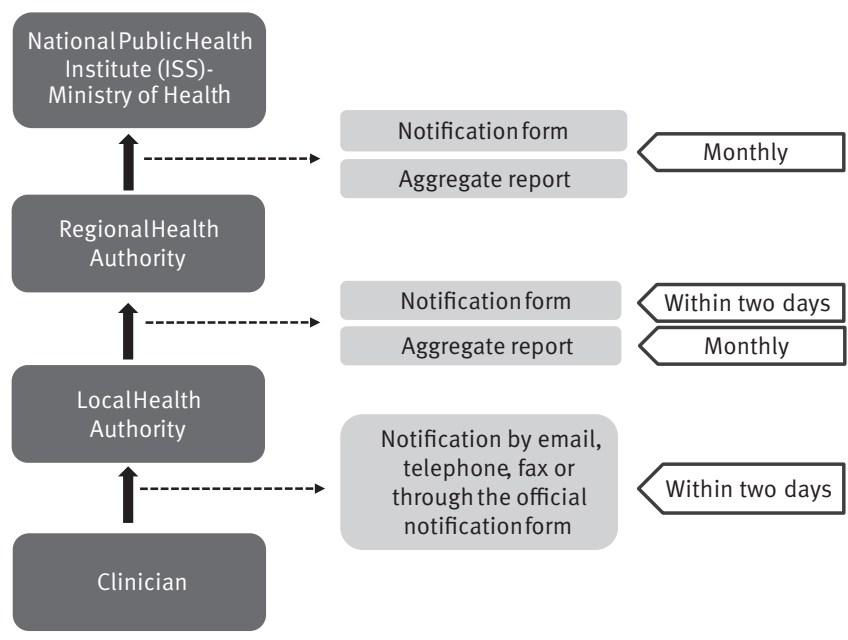

measured in Italy; in 2008, an epi-cluster survey conducted in 18 of the 21 Italian regions, found that rubella vaccine coverage was $75 \%$ in 16 year-old adolescents [7].

The national elimination plan recommends strengthening surveillance of rubella and congenital rubella cases. In Italy, postnatal rubella has been a notifiable disease since 1934, within a statutory surveillance system including 46 other infectious communicable diseases [8]; however, this system does not collect information on pregnancy status or on congenital rubella cases.

A national surveillance system for congenital rubella and rubella in pregnancy was implemented in 2005 [9]. This system is mandatory, passive, case-based and relies on reporting by clinicians (it is not laboratory based). Data flow is shown in Figure 1. Clinicians must notify suspected cases within two days to the local health authorities, who in turn are responsible for case investigations and monitoring newborns and pregnancy outcomes over time. Separate notification forms are used for congenital rubella and rubella infections in pregnancy and the notification form for congenital rubella also includes a section regarding the mother's history. Forms are forwarded to the regional health authorities who in turn send monthly reports to the Ministry of Health and the National Public Health Institute (Istituto Superiore di Sanità, ISS). Individual data are collected in a central database at the ISS and are regularly analysed. Case classifications are periodically updated based on follow-up data received by local health authorities [10]. A cross-check between the national database and regional archives of statutory notifications is performed yearly.
Surveillance systems for congenital rubella are active in 28 of 29 European Union (EU)/European Economic Area countries that participated in a survey conducted by the European Centre for Disease Prevention and Control (ECDC) in 2012 [11] and information on rubella infections in pregnancy was collected in 25 countries [11]. Although congenital rubella is notifiable at European level, incidence data are not collected by ECDC. They are reported from European countries to the WHO Regional Office for Europe through the WHO/ United Nations Children's Fund Joint Reporting Form and are made available on the WHO website on a yearly basis [12]. However, congenital rubella is not included in the list of vaccine-preventable diseases currently monitored at European level by ECDC through TESSy (The European Surveillance System).

In this paper we analyse Italian national surveillance data for congenital rubella and rubella infection in pregnancy from 2005 to 2013 , in order to monitor progress towards congenital rubella elimination and provide public health recommendations. Additionally, we discuss strengths and weaknesses of the surveillance system. Given the regional elimination goal, these data may be helpful to other public health actors in Europe.

\section{Methods}

\section{Congenital rubella infections}

We carried out a descriptive analysis of congenital rubella cases reported to the national surveillance system from 1 January 2005 to 31 December 2013. We classified cases as probable or confirmed according to the 2012 EU case definition for congenital rubella [13]. Cases for whom information was insufficient to confirm or exclude the diagnosis were excluded from the analysis. We calculated the incidence of congenital rubella by year and region, including confirmed and probable cases.

We described newborns with congenital rubella infection in terms of median gestational age, median weight at birth, sex, nationality and clinical manifestations. We also calculated the proportion of cases that satisfy the clinical criteria for CRS [13,14], that is (i) at least two of the category A conditions; or (ii) one category $A$ and one category B condition (where category A conditions include cataract, congenital glaucoma, congenital heart disease, loss of hearing and pigmentary retinopathy, and those in category $B$ include purpura, splenomegaly, microcephaly, developmental delay, meningo-encephalitis, radiolucent bone disease, jaundice that begins within 24 hours after birth).

In order to compare the incidence with the target of less than one case per 100,000 live births, we calculated the incidence of congenital rubella using the WHO Regional Office for Europe case definition (clinical CRS, epidemiologically linked CRS and laboratory-confirmed CRS) [14], which does not fully overlap with the EU case definition. The difference relates to asymptomatic 
Congenital rubella infections $(\mathrm{n}=75)$ and rubella infections in pregnancy $(\mathrm{n}=160)$ reported by year and case classification, Italy, 2005-13

\begin{tabular}{|c|c|c|c|c|c|c|c|}
\hline \multirow{2}{*}{ Year } & \multicolumn{3}{|c|}{ Congenital rubella ${ }^{a}$} & \multicolumn{4}{|c|}{ Rubella in pregnancy } \\
\hline & Probable & Confirmed & Total & Possible & Probable & Confirmed & Total \\
\hline 2005 & 1 & 2 & 3 & 0 & 0 & 6 & 6 \\
\hline 2006 & 1 & 0 & 1 & 0 & 0 & 1 & 1 \\
\hline 2007 & 1 & 2 & 3 & 1 & 0 & 4 & 5 \\
\hline 2008 & 0 & 29 & 29 & 0 & 1 & 76 & 77 \\
\hline 2009 & 3 & 10 & 13 & 0 & 0 & 7 & 7 \\
\hline 2010 & 0 & 2 & 2 & 0 & 0 & 5 & 5 \\
\hline 2011 & 0 & 2 & 2 & 0 & 0 & 4 & 4 \\
\hline 2012 & 1 & 18 & 19 & 3 & 7 & 40 & 50 \\
\hline 2013 & 0 & 3 & 3 & 0 & 1 & 4 & 5 \\
\hline Total & 7 & 68 & 75 & 4 & 9 & 147 & 160 \\
\hline
\end{tabular}

Cases were classified according to the 2012 European Union congenital rubella case definition [13].

b Cases were classified according to a modified version [10] of the 2012 European Union rubella case definition [13], that includes among the laboratory criteria for case confirmation a positive rubella IgM result supported by a rubella-specific IgG avidity test showing low avidity.

congenital infections. In particular, an infant born to a mother with confirmed rubella in pregnancy, with laboratory confirmation of infection but no rubella defects is classified as a confirmed case of congenital rubella according to the EU case definition, while the WHO Regional Office for Europe excludes cases without at least one Group A clinical condition [14]. Therefore, we excluded asymptomatic laboratory-confirmed cases to calculate incidence according to the WHO regional case definition.

In order to compare temporal trends of rubella and congenital rubella, we also calculated the incidence of postnatal rubella cases reported to the statutory surveillance system for communicable infectious diseases during 2005 to 2013. Data on postnatal rubella cases are collected in a central database at the Ministry of Health.

\section{Rubella infections in pregnancy}

We also carried out a descriptive analysis of rubella infections in pregnancy reported to the national surveillance system during 2005 to 2013. Reported cases included: (i) those notified through the notification form for rubella infection in pregnancy; and (ii) those whose data was obtained from the newborn's notification form (from the section regarding the mother's history), if mother's infection had not been previously notified.

We described cases in terms of median age at infection, nationality, parity, pregnancy trimester of infection, vaccination status, pre-pregnancy testing for rubella susceptibility and clinical manifestations.

Cases were classified as possible, probable or confirmed according to a modified version [10] of the 2012
EU rubella case definition [13], which includes among the laboratory criteria for case confirmation a positive rubella IgM result supported by a rubella-specific IgG avidity test showing low avidity. This criteria was added because when rubella infection is suspected during pregnancy, confirmation of a positive rubella IgM result (e.g. a rubella-specific IgG avidity test) is required [13].

\section{Pregnancy outcomes}

We matched data on congenital rubella cases and rubella infections in pregnancy (archived in two separate databases) in order to link pregnant women with their babies. We classified outcomes of pregnancy as live birth (infected, not infected or unknown state of infection), voluntary abortion, miscarriage and stillbirth. We also calculated the proportion of infected women who were lost to follow-up (for whom pregnancy outcome is unknown) and the proportion of infants, born to mothers with a possible, probable, or confirmed infection, who we were unable to classify either because they were lost to follow-up or because of insufficient data.

\section{Statistical analysis}

We summarised categorical variables using frequencies and proportions, and continuous variables as median and range. We used a chi-squared test or Fisher's exact test to compare proportions. We defined statistical significance as a $p$ value of $<0.05$.

For calculating the incidence of congenital rubella infections and CRS, we used the number of live births of each year (2005-13) obtained from the Italian National Institute of Statistics (ISTAT) [15]. For calculating the incidence of rubella cases reported to the statutory surveillance system for infectious diseases, we used 


\section{FIGURE 2}

Incidence of congenital $^{\mathrm{a}}(\mathrm{n}=75)$ and postnatal rubella cases $^{\mathrm{b}}(\mathrm{n}=8,421)$ and number of cases of rubella in pregnancy $(\mathrm{n}=160)^{\mathrm{c}}$, Italy, 2005-13

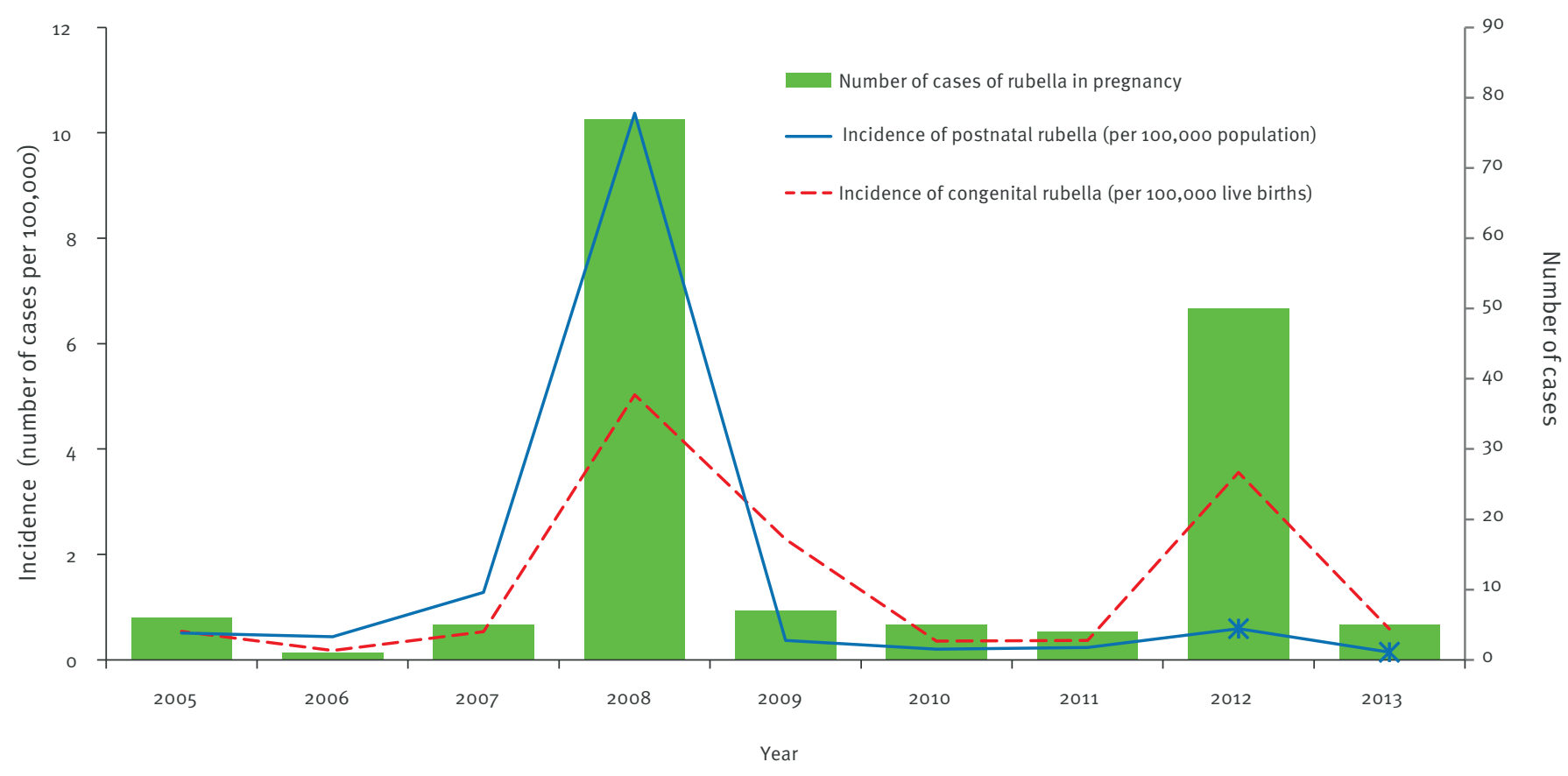

The data for incidence of postnatal rubella in 2012 and 2013 (marked with a cross) are provisional, due to the ongoing implementation of a web-based surveillance system for infectious diseases in Italy.

a Cased were classified according to the 2012 European Union case definition for congenital rubella [13].

b Cases were classified according to clinical criteria for rubella [8].

c Cases were classified according to a modified version [10] of the 2012 European Union rubella case definition [13], that includes among the laboratory criteria for case confirmation a positive rubella IgM result supported by a rubella-specific IgG avidity test showing low avidity.

the resident population data of each year (2005-13) obtained from ISTAT [15]. Statistical analysis was performed using Epi Info software version 3.5.4.

\section{Results}

\section{Congenital rubella}

A total of 75 congenital rubella infections (7 probable and 68 confirmed cases) were reported during 2005 to 2013, according to the 2012 EU congenital rubella case definition [13] (Table 1). We received an additional 59 reports of suspected cases who could not be classified because the available information was insufficient. These were excluded from the analysis.

The median birth weight of 67 of the cases for whom information was available was 2,710 g (range: 9134,330); 49/75 were male and 5/70 were born to foreign mothers. The median gestational age of cases was 38 weeks (range: 29-42) and 15/64 were born before the 37 th week of pregnancy.

Information on clinical manifestations was available for 73 cases. Of them, 16 were asymptomatic, whereas 57 had at least one clinical manifestation. Among these 57 symptomatic cases, 37 newborns satisfied the clinical criteria for CRS $[13,14], 17$ had only one group A condition and three had at least one group $B$ condition (but no group $A$ conditions). The most frequently reported condition was congenital heart disease $(n=41)$, followed by loss of hearing $(n=26)$, jaundice within 24 hours of birth $(n=18)$, meningo-encephalitis $(n=11)$, cataract $(n=12)$, microcephaly $(n=10)$, splenomegaly $(n=8)$, developmental delay $(n=7)$, purpura $(n=6)$, and pigmentary retinopathy $(n=1)$. A total of 20 cases had multiple defects involving the heart, hearing or vision.

The national annual mean incidence in the years studied was 1.5 per 100,000 live births (mean annual number of live births in Italy: 553,389), including probable and confirmed cases according to the 2012 EU case definition. Two incidence peaks of congenital rubella infections occurred in 2008 and 2012, with an incidence of 5.0 and 3.6 per 100,000 newborns, respectively (Figure 2).

Statutory notifications of cases of postnatal rubella also experienced a peak in 2008 and there was a slight increase in the number of reported cases in 2012 


\section{FIGURE 3}

Annual mean incidence of congenital rubella infections by region, including probable and confirmed cases ${ }^{a}$, Italy, 2005-13 $(\mathrm{n}=75)$

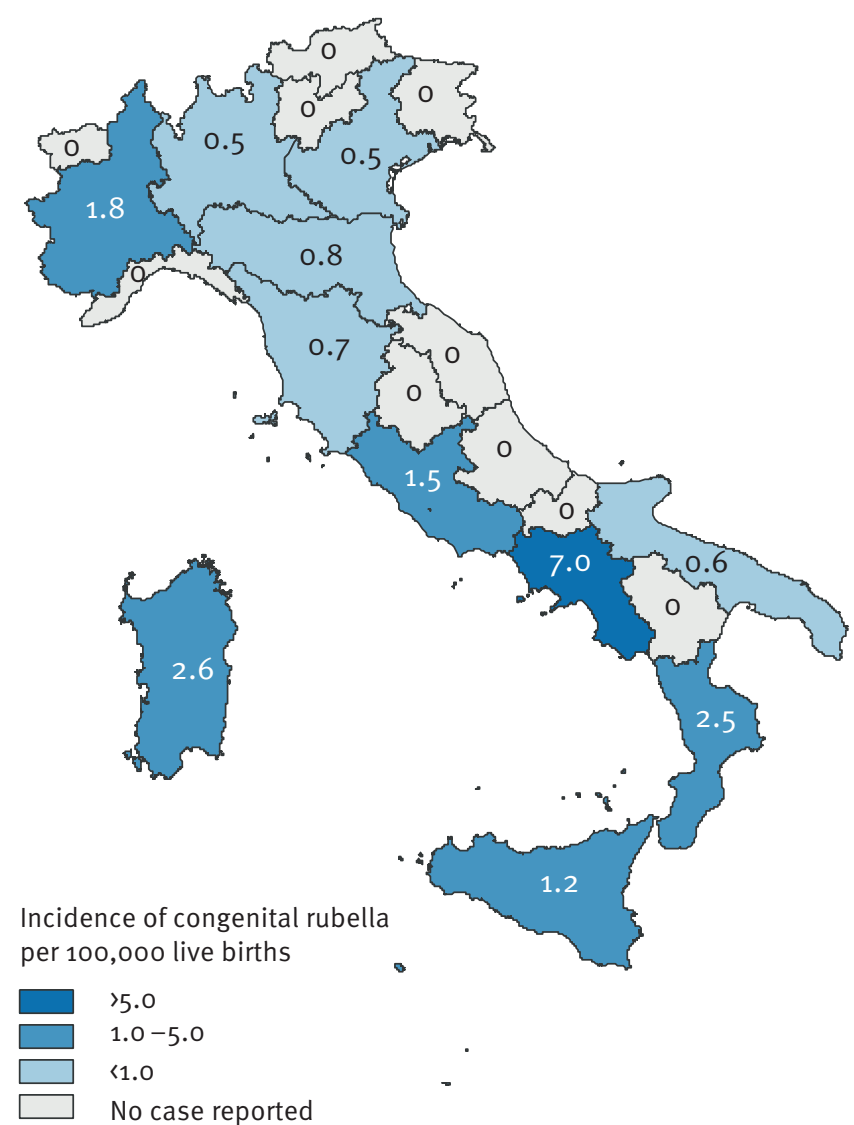

a According to the 2012 European Union congenital rubella case definition [13].

(provisional data) (Figure 2). During 2005 to 2013, a total of 8,421 cases of postnatal rubella were notified, with a median age of cases of 17.5 years (range: $0-88$ years).

Of the 21 regions of Italy, 11 reported congenital rubella cases, with regional annual mean incidences (confirmed and probable cases) in the years studied varying from 0.5 to 7.0 per 100,000 live births (Figure 3).

Most cases of congenital rubella reported in 2012 $(16 / 19)$ were notified by a single region in southern Italy (incidence of $29.2 / 100,000$ live births). In the same year, this region also reported 161 postnatal rubella cases, representing $46 \%$ of national cases $(n=353)$.

The national annual mean incidence of congenital rubella calculated according to the WHO case definition was 1.1 per 100,000 live births. In 2008, 2009 and 2012, it exceeded the threshold fixed by WHO to reach elimination $(2.9,1.8$ and 3.2 case per 100,000 live births respectively).

\section{Rubella infections in pregnancy}

Overall, 160 rubella infections in pregnancy were reported, of whom 147 were classified as confirmed, nine as probable and four as possible cases according to the modified version [10] of the 2012 EU rubella case definition [13] (Table 1). An additional 105 reports were unclassified because of incomplete data; these were excluded from the analysis.

The median age of the 107 cases for whom information was available was 26 years (range: 16-46). Of these, 23 were foreign-born.

Only three women reported being vaccinated against rubella, but this information was documented in a vaccination card for only one of the cases; the woman had received two vaccine doses in her country of origin, at the age of three and 13 years. However, she was found to have been susceptible to the infection at preconception screening.

Of the 107 cases for whom information on gestational age at infection was available, 45 acquired rubella during the first trimester of pregnancy; 69/148 were multiparous. Only 38/126 women had had a rubella antibody test before pregnancy; among them, 32 stated that they had been found to be susceptible, three were immunised and the test result was missing for three women (Table 2).

Characteristics of Italian and foreign-born infected women were similar but the proportion of multiparous women was significantly higher among the latter, compared with Italian women (68.2 vs $43.2, \mathrm{p}=0.0304$ ).

Notification forms of 51 of the 160 women with rubella infections in pregnancy were not received. In these 51 cases, information on demographic characteristics, clinical manifestations (reported in Table 2) and laboratory results were obtained from the notification forms of suspected congenital rubella of their babies (from the section regarding the mother's history). It indicates that in a large proportion of cases (32\%), the infection of the mother had not been reported during pregnancy and information was collected after delivery.

\section{Pregnancy outcomes}

Among the 160 women who acquired rubella during pregnancy (two sets of twins were included for this analysis, giving a total of 162 pregnancies), there were 29 uninfected and 62 infected newborns (4 probable and 58 confirmed congenital rubella cases according to EU case definition for congenital rubella). Of the 62 infected newborns, 46 had clinical manifestations (28 of them satisfied the clinical criteria for CRS, 16 had only one group A condition and two had at least one group $B$ condition) and 16 were asymptomatic. Overall, 24 newborns were not classified because of incomplete information $(n=19)$ or loss to follow-up $(n=5)$. 
A total of 31 voluntary abortions, one stillbirth and one spontaneous abortion were also recorded. Pregnancy outcome was unknown for 14 women who were lost to follow-up (Table 3).

\section{Discussion}

The data presented show that the national incidence of congenital rubella during the two epidemic peaks in 2008 and 2012 exceeded the target of less than one per 100,000 live births needed to reach elimination [2]. The incidence was below the WHO threshold in 2013 and provisional data indicate lower values in 2014 (with only one case of congenital rubella reported). However, it is known that rubella infection occurs in epidemic cycles [16] and elimination has not yet been achieved in Italy.

Underestimation of congenital rubella cases is likely for several reasons. Firstly, we found a high percentage of cases lost to follow-up and of unclassified cases. In particular, in $23 \%(38 / 162)$ of cases of infection in pregnancy, the pregnancy outcome was unknown or the newborn was not monitored for final case classification. Additionally, we received 59 reports of suspected congenital rubella cases and 105 reports of suspected rubella infections in pregnancy that could not be classified because of incomplete data, which were excluded from the analysis. Secondly, information on aborted fetuses and stillbirths was not available and it is likely that at least some were infected. Thirdly, the proportion of cases with hearing loss and cataracts is lower than that reported in literature $[16,17]$ and the proportion of cases with congenital heart diseases is higher [17], suggesting incomplete detection of milder cases.

Incidence calculated using the WHO case definition was obviously lower than that using the EU case definition because the former excluded asymptomatic laboratory-confirmed cases. In the framework of the elimination goal, the adoption of a common case definition would facilitate the evaluation of immunisation programmes.

Monitoring of suspected congenital rubella infections over time represents a critical aspect of the surveillance system, because a long follow-up is necessary to definitively classify cases. Laboratory confirmation of congenital infection is not always possible at birth (for instance, when infants are rubella-specific IgM negative at birth, a decrease in rubella-specific IgG levels by the age of 6-12 months allows the infection to be excluded) and also clinical manifestations are not necessarily present at birth.

Pregnancy outcomes of mothers with possible, probable or confirmed rubella infection during pregnancy also need to be monitored in order to detect congenital infections, including spontaneous or voluntary abortions or stillbirths that may occur if the infection is acquired in early pregnancy. No other sources of data for detecting abortions or stillbirths due to rubella infection are available in Italy.

Data from surveillance of rubella infections in pregnancy show that notification forms were not available for $32 \%(51 / 160)$ of the mothers; for these women, data were obtained from notification forms of their newborns, confirming underestimation of cases. Whenever information on mothers is obtained after delivery, it is not possible to obtain laboratory test results and, consequently, to correctly classify cases in a timely manner.

Several actions have been undertaken in Italy to improve the surveillance of congenital rubella and rubella infections in pregnancy. Firstly, at the end of 2013, the Ministry of Health disseminated national recommendations $[10]$ to reinforce the surveillance system. The EU case definition for congenital rubella was adopted, a case definition for rubella in pregnancy was

\section{TABLE 2}

Demographic and clinical characteristics of women who acquired rubella during pregnancy, Italy, 2005-13 (n = 160)

\begin{tabular}{|c|c|c|}
\hline Characteristic & Data & $\begin{array}{l}\text { Number of } \\
\text { cases (\%) }\end{array}$ \\
\hline $\begin{array}{l}\text { Median age at infection } \\
(n=107)\end{array}$ & 26 (range: $16-46)$ & NA \\
\hline \multirow{2}{*}{$\begin{array}{l}\text { Country of birth } \\
(n=159)\end{array}$} & Italy & $136(85.5)$ \\
\hline & Foreign country & $23(14.5)$ \\
\hline \multirow{3}{*}{$\begin{array}{l}\text { Trimester of pregnancy } \\
\text { at time of infection } \\
(n=107)\end{array}$} & First & $45(42.1)$ \\
\hline & Second & $41(38.3)$ \\
\hline & Third & $21(19.6)$ \\
\hline \multirow{2}{*}{$\begin{array}{l}\text { Vaccination status } \\
(n=127)\end{array}$} & Vaccinated & $3(2.4)$ \\
\hline & Unvaccinated & $124(97.6)$ \\
\hline \multirow{4}{*}{$\begin{array}{l}\text { Previous pregnancies } \\
(n=148)\end{array}$} & 0 & $79(53.4)$ \\
\hline & 1 & $34(23.0)$ \\
\hline & 2 & $25(16.9)$ \\
\hline & $\geq 3$ & $10(6.8)$ \\
\hline \multirow{2}{*}{$\begin{array}{l}\text { Rubella antibody } \\
\text { testing before } \\
\text { pregnancy } \\
(n=126)\end{array}$} & Performed & $38(30.2)$ \\
\hline & Not performed & $88(69.8)$ \\
\hline \multirow{3}{*}{$\begin{array}{l}\text { Clinical manifestations } \\
(n=148)\end{array}$} & $\begin{array}{c}\text { Clinical criteria } \\
\text { EU case definition } \\
\text { fully } \text { met }^{\mathrm{a}}\end{array}$ & $76(51.4)$ \\
\hline & $\begin{array}{l}\text { Clinical criteria } \\
\text { EU case definition } \\
\text { partially } \text { met }^{b}\end{array}$ & $46(31.1)$ \\
\hline & $\begin{array}{c}\text { Asymptomatic, } \\
\text { laboratory confirmed }\end{array}$ & $26(17.6)$ \\
\hline
\end{tabular}

EU: European Union; NA: not applicable.

a Clinical criteria for 2012 EU rubella case definition [13] fully met: sudden onset of generalised maculo-papular rash AND at least one of the following: cervical adenopathy, sub-occipital adenopathy, post-auricular adenopathy, arthralgia, arthritis.

b Clinical criteria for 2012 EU rubella case definition [13] partially met: maculo-papular rash OR cervical adenopathy OR sub-occipital adenopathy OR post-auricular adenopathy OR arthralgia OR arthritis. 
Pregnancy outcomes of rubella infections acquired in pregnancy by case classification of mothers, Italy, 2005-13 ( $\mathrm{n}=162)^{\mathrm{a}}$

\begin{tabular}{|c|c|c|c|c|}
\hline \multirow{2}{*}{ Pregnancy outcome } & \multicolumn{3}{|c|}{ Case classification of mothers $^{b}$} & \multirow{2}{*}{ Number $(\%)$} \\
\hline & Possible & Probable & Confirmed & \\
\hline Newborns infected & 3 & 4 & 55 & $62(38.3)$ \\
\hline Newborns not infected & 1 & 3 & 25 & $29(17.9)$ \\
\hline Newborns with unknown state of infection & 0 & 1 & 23 & $24(14.8)$ \\
\hline Voluntary abortion & 0 & o & 31 & $31(19.1)$ \\
\hline Spontaneous abortion & 0 & o & 1 & $1(0.6)$ \\
\hline Stillbirth & 0 & o & 1 & $1(0.6)$ \\
\hline Mothers lost to follow-up & o & 1 & 13 & $14(8.6)$ \\
\hline
\end{tabular}

\footnotetext{
162 outcomes (including two sets of twins) from 160 infected mothers.

b Cases were classified according to a modified version [10] of the 2012 European Union rubella case definition [13], that includes, among the laboratory criteria for case confirmation, a positive rubella IgM result supported by a rubella-specific IgG avidity test showing low avidity.
}

introduced (a modified version of the EU case definition for rubella) and the notification forms were modified, adding new variables to be collected, such as importation status (endemic, imported, import-related cases) and genotyping, which are critical for assessing the elimination of endemic rubella. Additionally, systematic procedures for monitoring infected pregnant women (until the end of pregnancy) and their newborns (at birth, 1, 6, 12, 18 and 24 months) were introduced. However these recommendations have not yet been implemented in all local health authorities. Secondly, an integrated surveillance system for measles and rubella was implemented at the national level in 2013 [18], requiring laboratory investigation of suspected cases of rubella and web-based reporting of cases. It could facilitate the early detection of rubella infections in pregnancy and encourage a timely follow-up. Thirdly, in order to assess under-reporting, an evaluation of the completeness of reporting to the surveillance system is being conducted at the national level, by analysing hospital discharge records for 2010 to 2014 to identify cases discharged with a diagnosis of congenital rubella (International classification of diseases, ninth revision, clinical modification (ICD-9-CM) code 771.0) [19].

In the Puglia region, hospital discharge records for 2003 to 2011 were analysed to identify ICD-9-CM codes 647.5 (rubella in pregnancy) and 771.0 (congenital rubella) and individual records of identified cases were retrieved [20]. Delivery-assistance certificate registries were also analysed to retrieve clinical histories of mothers of babies with CRS. One CRS, two congenital asymptomatic rubella infections and four suspected congenital rubella cases were identified, who were not included in the national surveillance database.

Data from laboratories could be an alternative source to assess under-reporting. However, in Italy, diagnostic testing for rubella infection (also in pregnancy) is often performed in private laboratories and building a network involving all these laboratories would be difficult to implement.

In order to prevent congenital rubella, susceptible women need to be identified and vaccinated before pregnancy. The Italian national elimination plan [3] has highlighted the need to reduce to below $5 \%$ the percentage of women of childbearing age who are susceptible to rubella. In Italy, the last national seroprevalence survey was conducted in 2004 , showing that $11 \%$ of women aged $15-19$ years and $8 \%$ aged $20-39$ years were susceptible [21]. This study was conducted before preventive activities were implemented at the national level; however, some local studies published in 2012 have found a continued high percentage of women of childbearing age at risk of rubella infection, varying from $8 \%$ to $14 \%[22,23]$.

Rubella pre-conception screening is substantially underused in Italy, although it is available free of charge [24]. Data from the Progressi delle Aziende Sanitarie per la Salute in Italia [Progress by Local Health Units towards a Healthier Italy] (PASSI) Italian behavioural risk factor surveillance system showed that in $2010,38 \%$ of $11,45018-49$ year-old women were not aware of their rubella immunisation status [25]. Additionally, a rubella seroprevalence study conducted in 2006 to 2007, targeting a group of pregnant women who had been referred to a prenatal clinic in southern Italy, found that only $55 \%$ of 500 pregnant women had undergone screening before pregnancy [23]. In our study, only $30 \%$ of infected women had verified their rubella immunity status before pregnancy and most of those found to be susceptible were not vaccinated. According to the national elimination plan, the rubella immunisation status of women of childbearing age should be evaluated whenever possible (e.g. concomitantly with human papilloma virus vaccination, at the 10-yearly anti-diphtheria-tetanus-pertussis booster 
dose, at the first Pap test screening visit, at the first vaccination of their newborns, at the first contact of immigrant women with healthcare services), by checking vaccination cards or measuring rubella-specific IgG antibodies, and susceptible women should be promptly offered vaccination. Systematic reporting by laboratories of any negative rubella antibody results to vaccination services could be useful for an active search of seronegative women.

We found that a large proportion (47\%) of infected women were multiparous, indicating that they had missed the opportunity to be vaccinated after previous pregnancies. The 2006-07 seroprevalence study mentioned above also found a high proportion of multiparous women: of 71 pregnant women $(14.2 \%$ of the overall sample) susceptible to rubella, $33.8 \%$ had had at least one previous pregnancy [23].

About $14 \%$ of infected mothers in our study were not Italian-born, consistent with the rate of newborns from foreign-born mothers during 2005 to 2013 in Italy (9.4-15.1\%) [15]. The significantly higher proportion of multiparous women among foreign-born women, compared with those who were Italian, suggests that particular attention needs to be given to this population group, as they may have limited access to healthcare services because of language and culture.

A serosurvey of 2,385 pregnant women, carried out in a region of northern Italy during 2008 to 2013 found that $11.7 \%$ of non-Italian women were seronegative for rubella-specific IgG and this proportion was higher than that of Italian women $(6.2 \%, p<0.01)$ [22]. A serosurvey of 489 immigrant women, carried out during 2008 to 2009 in a town in southern Italy, found $17.8 \%$ seronegative women; $67 \%$ of the overall sample declared having no knowledge of rubella as a potential harm to fetus if the infection is contracted during pregnancy [26].

It is strongly advised to vaccinate all susceptible pregnant women with MMR vaccine during the post-partum (or post-abortum) period, in order to prevent the infection during a future pregnancy. The high proportion of multiparous women among reported cases of rubella in pregnancy shows that post-partum vaccination in Italy is not a routine procedure. Several post-partum vaccination strategies have been proposed in the national elimination plan: (i) immunisation in hospital before discharge; (ii) active call and immunisation at the public vaccination service (the hospital should forward the list of the susceptible women to the immunisation services); and (iii) immunisation at the public vaccination service concomitantly with the first vaccination of the newborn. A qualitative study, carried out in Australia in 2012 to explore the reasons for low maternal vaccine uptake, found that the incorporation of rubella susceptibility detection and maternal vaccination into standard care through a structured process was an important facilitator for immunisation uptake and offered an effective template for other perinatal management, such as pertussis and influenza vaccination [27].

More intensive regional approaches are needed in Italy, as variability of congenital rubella incidence was detected among the regions. In fact, the peak that occurred in 2012 was mostly attributable to a single southern region that notified $84 \%$ of all nationally reported cases, with a yearly incidence of $29.2 / 100,000$ live births. A high rate of susceptible women of childbearing age is one explanation for the high incidence in this region, which historically had lower MMR vaccination coverage in children, compared with other regions [28]. The presence of a regional registry of perinatal infections, active since 1996 in this region, may have contributed to improved reporting of cases [29]. Collection of MMR vaccination coverage of adolescents and adults and seroprevalence data would facilitate regional evaluations.

\section{Conclusion}

Several actions have been implemented in Italy to strengthen surveillance of congenital rubella and rubella in pregnancy; however, further efforts are needed to ensure that these activities are implemented across the country. In particular, systematic procedures for the follow-up of infected children and mothers should be adopted in all regions.

In order to protect women of childbearing age from rubella infection, routine rubella antibody screening before pregnancy (which is recommended in Italy and offered free of charge) and vaccination of susceptible women, including post-partum and post-abortum vaccination, should be strongly promoted by clinicians.

Healthcare workers (general practitioners, paediatricians, gynaecologists and other specialists) should be sensitised and trained, both for enforcing notification procedures and for evaluating women's rubella immunisation status as a priority task during healthcare visits. Also, information campaigns for the general population are needed to increase awareness of the risk of acquiring rubella infection during pregnancy. Particular attention needs to be focused in high-incidence regions.

Finally, high two-dose MMR vaccination coverage of children should be maintained $(\geq 95 \%)$ in order to interrupt viral circulation among the population.

raised among clinicians about the risk of leptospirosis exposure among these groups.

Acknowledgments

Surveillance of congenital rubella and rubella in pregnancy is carried out with financial support of the Italian Ministry of Health - CCM. We wish to thank all the regional and local contact points for rubella, the clinicians and the laboratories that routinely contribute to the notification, investigation, data collection and monitoring of cases. We wish also 
to thank Wilma Buffolano, coordinator of the Registry of Perinatal Infections and the Network of the Delivery Clinics (RePuNaRC) for Campania Region, for her helpful support in tracing and following up cases in her region.

\section{Conflict of interest}

None declared.

\section{Authors' contributions}

Cristina Giambi coordinated surveillance activities for congenital rubella and rubella in pregnancy at the nationa level, analysed the data, interpreted the results, drafted and edited the manuscript. Antonietta Filia, Maria Cristina Rota and Silvia Declich coordinated surveillance activities, interpreted the results and critically revised the manuscript. Antonino Bella and Martina Del Manso analysed the data, interpreted the results and critically revised the manuscript. Gloria Nacca entered individual reports and follow-up information in the national database. Elvira Rizzuto provided data on postnatal rubella cases and critically revised the manuscript. Regional contact points for rubella coordinated surveillance activities at the regional level and critically revised the manuscript.

\section{Regional contact points for rubella}

Di Giacomo M (Abruzzo); Locuratolo F (Basilicata); Natter B (PA Bolzano); Mignuoli A (Calabria); Pizzuti R (Campania); Pascucci MG, Moschella L (Emilia-Romagna); Gallo T., Braida C. (Friuli Venezia Giulia); Vitagliano A, Guerra M (Lazio); Cremonesi I (Liguria); Coppola L, Piatti A (Lombardia); Fiacchini D, Damiani N (Marche); Bagnoli C, Ponzio GV (Molise); Ferrara L (Piemonte); Prato R, Cappelli MG (Puglia); Salotto M (Sardegna); Palermo M (Sicilia); Balocchini E, Gallicchio S (Toscana); Carraro V (PA Trento); Sudano L (Valle d’Aosta); Russo F, Zanella R (Veneto); Tosti A (Umbria).

\section{References}

1. Duszak RS. Congenital rubella syndrome--major review. Optometry. 2009;80(1):36-43. http://dx.doi.org/10.1016/j. optm.2008.03.006 PMID:19111256

2. World Health Organization (WHO) Regional Office for Europe. Eliminating measles and rubella. Framework for the verification process in the WHO European Region. Copenhagen: WHO Regional Office for Europe; 2012. Available from: http://www. euro.who.int/_data/assets/pdf_file/0005/156776/e96153Eng-final-version.pdf

3. Ministero della Salute [Italian Ministry of Health]. Piano nazionale per l'eliminazione del morbillo e della rosolia congenita 2010-2015. [National Plan for the elimination of measles and congenital rubella 2010-2015]. Rome: Ministero della Salute; 2011. [Accessed 4 Nov 2014]. Italian. Available from: http://www.salute.gov.it/imgs/C 17 pubblicazioni_1519_allegato.pdf

4. United States Centers for Disease Control and Prevention (CDC). National Center for Immunization and Respiratory Disease Division of Viral Diseases. CDC Report: Elimination of Measles, Rubella, and CRS. Documentation and verification of measles, rubella and congenital rubella syndrome elimination in the Region of the Americas. United States National Report, March 28, 2012. Atlanta, GA: CDC; 2012. Available from: http://www.cdc.gov/measles/downloads/Report-eliminationmeasles-rubella-crs.pdf

5. Italian Ministry of Health. Piano nazionale prevenzione vaccinale 2012-2014. [National Vaccination Prevention Plan 2012-2014]. Gazzetta Ufficiale [Official Bulletin]. 12 Mar 2012 no. 6o. [Accessed 4 Nov 2014]. Italian. Available from: http:// www.salute.gov.it/imgs/C 17 pubblicazioni_1721 allegato.pdf

6. Ministero della Salute [Italian Ministry of Health]. Malattie infettive e vaccinazioni. Coperture vaccinali. [Infectious diseases and vaccinations. Vaccine coverage]. Rome:
Ministero della Salute. [Accessed 4 Nov 2014]. Italian. Available from: http://www.salute.gov.it/malattielnfettive/ paginalnternaMenuMalattielnfettive.jsp?id=811\&menu=strum entieservizi

7. ICONA Working Group. ICONA 2008: Indagine di copertura vaccinale nazionale nei bambini e negli adolescenti. [ICONA 2008: national vaccination coverage survey among children and adolescents]. Rapporti ISTISAN. 09/29. Rome: Istituto Superiore di Sanità; 2009. [Accessed 4 Nov 2014]. Italian. Available from: http://www.iss.it/binary/publ/cont/o9_29 web.pdf

8. Ministero della Salute [Italian Ministry of Health]. Decreto ministeriale 15 dicembre 1990 - Sistema informativo delle malattie infettive e diffusive. [Ministerial decree 15 December 1990 - Informative system for infectious communicable diseases]. Rome: Ministero della Salute; 15 December 1990. Gazzetta Ufficiale [Official Bulletin]. 8 Jan 1991; no. 6. [Accessed 4 Nov 2014]. Italian. Available from: http://www. salute.gov.it/imgs/C_17_normativa_1357_allegato.pdf

9. Ciofi Degli Atti ML, Filia A, Verteramo R, Iannazzo S, Curtale $\mathrm{F}$, Masini L, et al. First cases of rubella infection during pregnancy detected by new reporting system in Italy. Euro Surveill. 2006;11(12): $\mathrm{pii}=2930$.

10. Ministero della Salute [Italian Ministry of Health]. Sorveglianza della rosolia congenita e dell'infezione da virus della rosolia in gravidanza alla luce del nuovo Piano Nazionale di Eliminazione del morbillo e della rosolia congenita 2010-2015. [Surveillance of congenital rubella and rubella infection in pregnancy according to the new National Plan for measles and congenital rubella elimination 2010-2015]. Rome: Ministero della Salute; 17 July 2013. [Accessed 4 Nov 2014]. Italian. Available from: http://www.trovanorme.salute.gov.it/norme/renderNormsanPd $f ?$ anno $=0 \&$ codLeg $=46583$ \& parte $=1 \% 20 \&$ serie $=$

11. European Centre for Disease Prevention and Control (ECDC). Survey on rubella, rubella in pregnancy and congenital rubella surveillance systems in EU/EEA countries. Stockholm: ECDC; 2013. Available from: http://ecdc.europa.eu/en/publications/ Publications/survey-rubella-pregnancy-congenitalsurveillance-systems-may-2013.pdf

12. World Health Organization (WHO). Rubella (crs) reported cases Reported incidence time series. Geneva: WHO. [Accessed 4 Nov 2014]. Available from: http://apps.who.int/immunization monitoring/globalsummary/timeseries/tsincidencecrs.html

13. European Commission. Commission Implementing Decision of 8 August 2012 (2012/506/EU) amending Decision 2002/253/ EC laying down case definitions for reporting communicable diseases to the Community network under Decision No 2119/98/EC of the European Parliament and of the Council. Official Journal of the European Union. Luxembourg: Publications Office of the European Union. 27.9.2012;L 262. Available from: http://eur-lex.europa.eu/LexUriServ/ LexUriServ.do?uri=0J:L:2012:262:0001:0057:EN:PDF

14. World Health Organization (WHO) Regional Office for Europe. Surveillance guidelines for measles, rubella and congenital rubella syndrome in the WHO European Region. Update December 2012. Copenhagen: WHO Regional Office for Europe; 2012. Available from: http://www.euro.who.int/_data/assets/ pdf_file/0018/79020/e93035-2013.pdf

15. Italian National Institute of Statistics (ISTAT). Popolazione residente, bilancio demografico e cittadinni stranieri.[Resident population, demographic balance and resident foreigners]. Rome: ISTAT. [Accessed 4 Nov 2014]. Available from: demo. istat.it

16. Plotkin SA, Reef SE. Rubella vaccine. In: Plotkin SA, Orenstein W, Offit P, editors. Vaccines. 5th ed.: Saunders Elsevier; 2008. p. 735-71.

17. Dontigny L, Arsenault MY, Martel MJ, Biringer A, Cormier J, Delaney M, et al.; Society of Obstetricians and Gyneacologist of Canada. Rubella in pregnancy. J Obstet Gynaecol Can. 2008;30(2):152-68. PMID:18254998

18. Ministero della Salute Italian Ministry of Health. Istituzione di un sistema di sorveglianza integrato per il morbillo e la rosolia alla luce del nuovo Piano Nazionale di Eliminazione del morbillo e della rosolia congenita 2010-2015. [Implementation of an integrated surveillance system for measles and rubella according to the new National Plan for measles and congenital rubella elimination 2010-2015]. Rome: Ministero della Salute; 20 February 2013. Italian. Available from: http://www. sitiappulolucana.it/images/pdf.scaricabili/PNEMoRc\%2020102015\%20completo.pdf

19. Centers for Disease Control and Prevention (CDC). International classification of diseases, ninth revision, clinical modification (ICD-9-CM). Atlanta, GA: CDC; 2010. [Accessed 4 Nov 2014]. Available from: http://www.cdc.gov/nchs/icd/icd9cm.htm

20. Cozza V, Martinelli D, Cappelli MG, Tafuri S, Fortunato F, Prato R. Further efforts in the achievement of congenita rubella syndrome/rubella elimination: alternative sources of 
information for retrospective case-finding, Puglia Region, Italy, 2003-2011. Hum Vaccin Immunother. 2015;11(1):220-4. http:// dx.doi.org/10.4161/hv.36154 PMID:25483539

21. Rota MC, Bella A, Gabutti G, Giambi C, Filia A, Guido M, et al.; Serological Study Group. Rubella seroprofile of the Italian population: an 8-year comparison. Epidemiol Infect. 2007;135(4):555-62. http://dx.doi.org/10.1017/ So950268806007400 PMID:17076939

22. De Paschale M, Manco MT, Paganini A, Agrappi C, Mirri P, Cucchi $G$, et al. Rubella antibody screening during pregnancy in an urban area of northern Italy. Infect Dis Rep. 2012;4(1)e17.

23. Calimeri S, Capua A, La Fauci V, Squeri R, Grillo OC, Lo Giudice D. Prevalence of serum anti-rubella virus antibodies among pregnant women in southern Italy. Int J Gynaecol Obstet. 2012;116(3):211-3. http://dx.doi.org/10.1016/j.ijg0.2011.10.029 PMID:22233835

24. Italian Ministry of Health. Aggiornamento del d.m. 6 marzo 1995 concernente l'aggiornamento del d.m. 14 aprile 1984 recante protocolli di accesso agli esami di laboratorio e di diagnostica strumentale per le donne in stato di gravidanza e a tutela della maternità. [Procedures to access to laboratory exams and instrumental methods of diagnosis for pregnant women and maternity's protection]. Rome: Ministero della Salute. Ministerial Decree 10 September 1998. Italian. Gazzetta Ufficiale [Official Bulletin]. 20 October 1998, no. 245.

25. Istituto Superiore di Sanità. La sorveglianza Passi. Rapporto nazionale Passi 2010: vaccinazione antirosolia. [PASSI surveillance. PASSI national report 2010: vaccination against rubella]. Italian. [Accessed 4 Nov 2014]. Available from: http://www.epicentro.iss.it/passi/rapporto2010/ R2010VaccinazioneAntirosolia.asp

26. Lo Giudice D, Capua A, La Fauci V, Squeri R, Grillo OC, Calimeri S. Congenital rubella syndrome and immunity status of immigrant women living in southern Italy: a crosssectional, seroepidemiological investigation. Travel Med Infect Dis. 2014;12(3):253-7. http://dx.doi.org/10.1016/j. tmaid.2014.01.003 PMID:24502919

27. Webb H, Street J, Marshall H. Incorporating immunizations into routine obstetric care to facilitate Health Care Practitioners in implementing maternal immunization recommendations. Hum Vaccin Immunother. 2014;10(4):1114-21. http://dx.doi. org/10.4161/hv.27893 PMID:24509790

28. ICONA Working Group. ICONA: Indagine nazionale sulla copertura vaccinale infantile. [ICONA: national survey on pediatric vaccination coverage]. Rapporti ISTISAN. 98/33. Rome: Istituto Superiore di Sanità; 1998. Italian.

29. Buffolano W, Lorenzo E, Lodato S, Parlato A, Pizzuti R, Onlus Rete Punti Nascita Regione Campania, Servizi Epidemiologia e Prevenzione ASL Regione Campania (SEP). Surveillance of congenital rubella: the Campania "Perinatal Infections" Registry experience. Bollettino epidemiologico nazionale (BEN) - Notiziario ISS. 2003;16(5). [Accessed 4 Nov 2014]. Available from: http://www.epicentro.iss.it/ben/2003/ maggio2003/4_en.htm 